ARTICLE

DOI: $10.1038 / s 41467-017-02215-7$

\title{
Electrical semiconduction modulated by light in a cobalt and naphthalene diimide metal-organic framework
}

Evandro Castaldelli (10 1,2, K.D.G. Imalka Jayawardena², David C. Cox², Guy J. Clarkson (1) ${ }^{3}$, Richard I. Walton ${ }^{3}$, Long Le-Quang ${ }^{4}$, Jerôme Chauvin ${ }^{4}$, S. Ravi P. Silva (i] ${ }^{2}$ \& Grégoire Jean-François Demets (i) ${ }^{1}$

Metal-organic frameworks (MOFs) have emerged as an exciting class of porous materials that can be structurally designed by choosing particular components according to desired applications. Despite the wide interest in and many potential applications of MOFs, such as in gas storage, catalysis, sensing and drug delivery, electrical semiconductivity and its control is still rare. The use and fabrication of electronic devices with MOF-based components has not been widely explored, despite significant progress of these components made in recent years. Here we report the synthesis and properties of a new highly crystalline, electrochemically active, cobalt and naphthalene diimide-based MOF that is an efficient electrical semiconductor and has a broad absorption spectrum, from 300 to $2500 \mathrm{~nm}$. Its semiconductivity was determined by direct voltage bias using a four-point device, and it features a wavelength dependant photoconductive-photoresistive dual behaviour, with a very high responsivity of $2.5 \times 10^{5} \mathrm{~A} \mathrm{~W}^{-1}$.

\footnotetext{
${ }^{1}$ Departamento de Química, FFCLRP, Universidade de São Paulo, Av. Bandeirantes 3900, Ribeirão Preto SP 14040-901, Brazil. ${ }^{2}$ Advanced Technology Institute, University of Surrey, Guildford Surrey GU2 7XH, UK. ${ }^{3}$ Department of Chemistry, University of Warwick, Coventry CV4 7AL, UK. ${ }^{4}$ CIRE/DCM Université de Grenoble Alpes, CS 40700, Grenoble Cedex 9, 38058, France. Correspondence and requests for materials should be addressed to E.C. (email: evandro.castaldelli@gmail.com) or to S.P.S. (email: s.silva@surrey.ac.uk) or to G.J.-F.D. (email: greg@usp.br)
} 
M

etal-organic frameworks (MOFs) are a class of robust, microporous, crystalline organic-inorganic hybrid materials that have received much attention due to their myriad of properties and applications as well as owing to their inexpensive starting materials and simple synthetic routes $^{1-3}$. MOFs may also feature large surface areas and inherit electronic and optical properties from their component metals and ligands. Despite the virtually infinite number of combinations between different ligands and metal centres, application of MOFs usually exploit their surface areas, mainly for gas adsorption ${ }^{4}$ and selective catalysis ${ }^{5}$. Their properties can also be tuned or altered depending on the characteristics of any adsorbed species and guest molecules 6,7 . These coordination polymers are normally insulating materials with only a fraction being designed for electronic semiconductivity ${ }^{8-10}$, and almost none display any electrical semiconductivity for practical purposes ${ }^{11}, 12$. Examples of redox activity ${ }^{13-16}$, photoactivity ${ }^{17}, 18$ and electronic semiconductivity ${ }^{19-21}$ in MOFs can be found in the literature, but to a more limited extent than those focused on catalysis or gas adsorption. Most notably, Dincă et al. developed a series of MOFs with intrinsic semiconductivity ${ }^{8-10}$, while Nishihara also developed a series of electroactive, photoactive and semiconductive nanosheets of $2 \mathrm{D}$ coordination polymers with then record-high conductivities $^{22-26}$. Another field where MOFs are currently being considered is solar cells, where they have been combined with well-known active semiconductors, such as $\mathrm{TiO}_{2}$ and carbon nanotubes ${ }^{27,28}$. Despite their current limitation as intrinsic semiconductors, great progress has been made in recent years in the field of MOFs for electronics and photonics ${ }^{29}$.

1,4,5,8-naphthalene diimides (NDIs) are a class of organic compounds that form air-stable n-type organic semiconductors as thin films with high charge mobilities ${ }^{30}$. Their unique electrochemical and optical properties make them attractive materials for electronic applications, such as active layers in transistors ${ }^{31}$ and solar cells $\mathrm{s}^{32}$, and as electron-transport layers in organic lightemitting devices ${ }^{33}$. Most of the electronic and optical properties of NDIs are governed by their frontier orbitals, mainly localised on the naphthalene core, and they usually experience small or minimal effects from their side chains ${ }^{34}$. In this sense, it is possible to choose suitable side chains to act as coordination points with no considerable influence on core properties. Redox-active
MOFs with NDI ligands have been developed featuring the typical radical anion $\left(\mathrm{NDI}^{-}\right)$and dianion $\left(\mathrm{NDI}^{2-}\right)$ species with interesting optical characteristics ${ }^{11}, 13,35$, and in-depth analysis has been carried out using computational methods ${ }^{36}$ and spectroelectrochemistry ${ }^{37}$. Their electron accepting nature may be exploited in extended networks to promote electron-hopping semiconduction between multivalent ionic metallic centres, such as $(\mathrm{Ru}, \mathrm{Fe}, \mathrm{Os} \text { or } \mathrm{Co})^{\mathrm{II} / \mathrm{III}}$ and others ${ }^{19}$. It is well known that NDIs may undergo light-induced reduction, forming radical anions and dianions, like negative polarons and bipolarons ${ }^{38}$.

Rationally combining NDI properties with a suitable metal may be key to yield semiconductive MOFs. The concept of efficient photoactive semiconducting MOFs by combining the stability, large surface areas and versatility of MOFs is crucial for the development of electrical and photoelectrical devices.

Here we report a unique, highly crystalline NDI-based MOF with semiconducting properties. We chose $\mathrm{Co}(\mathrm{II})$ as the metal centre to explore its suitable Pearson's hardness to $\mathrm{N}$-coordinated ligands, and to exploit the electronic structure and spectroscopic features it delivers ${ }^{39}, \quad N, N^{\prime}$-bis(4-pyridyl)-1,4,5,8-naphthalene diimide (NDI-py) as the primary ligand and terephthalic acid (TpA) as a supporting ligand. This framework is labelled MOFCoNDI-py-2, and its crystal structure and electronic properties are analysed in depth in order to understand its light sensitive electrical semiconductivity. Photocurrents and responsivities are assessed by direct measurements using a fabricated electronic device under a monochromatic light of different wavelengths. These results are evaluated in terms of anisotropy of semiconductivity and photoactivity, and are correlated with electronic absorption bands using a symmetry-adapted ligand field theory model.

\section{Results}

Synthesis of MOF-CoNDI-py-2. The compound was synthesised solvothermally in $\mathrm{N}, \mathrm{N}$-dimethylformamide (see 'Methods' section). MOF-CoNDI-py-2 forms purple leaf-like plates, which crystallised in the monoclinic space group $C 2 / c$ and the contents of the unit cell are shown in Fig. 1. The asymmetric unit contains two crystallographically independent high spin $\mathrm{Co}(\mathrm{II})$ atoms, Co (1) and $\mathrm{Co}(2)$, whose valence were identified by EPR

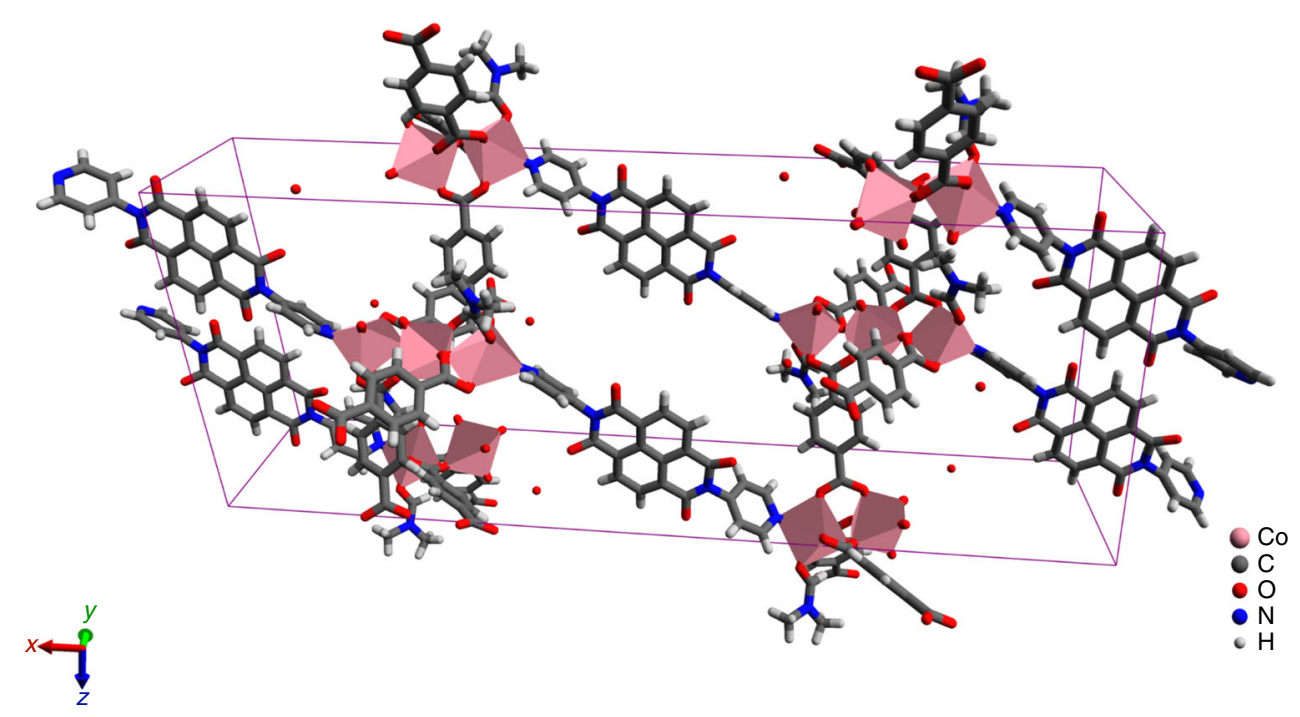

Fig. $1 \mathrm{~A}$ representation of MOF-CoNDI-py-2 crystal structure including its unit cell obtained by single-crystal XRD. Pink $=$ cobalt, grey $=$ carbon, white $=$ hydrogen, blue $=$ nitrogen and red $=$ oxygen. The unit cell belongs to a $C 2 / c$ monoclinic space group, water was modelled at $1 / 4$ occupancy and its hydrogens are not shown for clarity. The structure shows the NDI-py molecules oriented in a layered fashion. The distances between NDI-py entities are $9.43 \AA$ along the $c$-axis, $9.57 \AA$ along the $b$-axis and $11.10 \AA$ through the metal cluster 


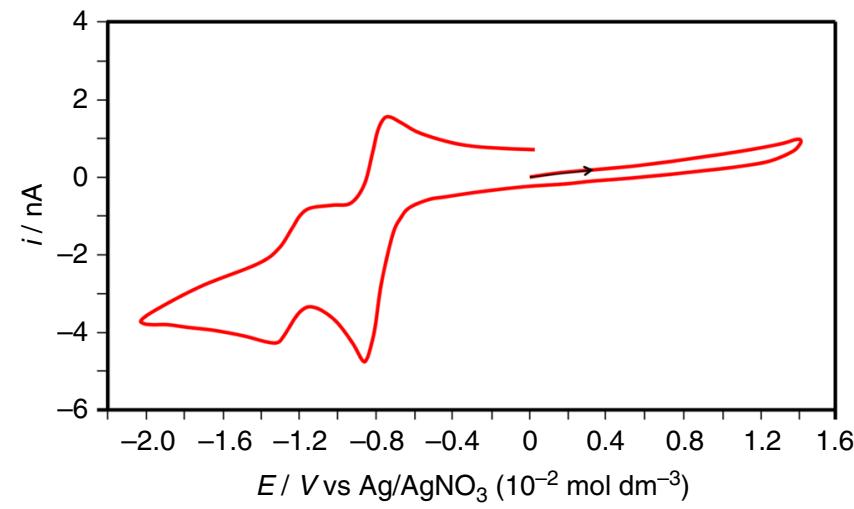

Fig. 2 Solid-state cyclic voltammetry of MOF-CoNDI-py-2 in MeCN. A cavity microelectrode was used as working electrode, a platinum wire as auxiliary and $\mathrm{Ag} / \mathrm{AgNO}_{3}\left(10 \mathrm{mmol} \mathrm{dm}{ }^{-3}, \mathrm{MeCN}\right)$ as reference, in a $0.1 \mathrm{~mol}$ $\mathrm{dm}^{-3}\left[\mathrm{~N}(n-\mathrm{Bu})_{4}\right]\left(\mathrm{PF}_{6}\right)$ solution in acetonitrile at $50 \mathrm{mV} \mathrm{s}^{-1}$. The voltammogram shows characteristic naphthalene diimide reduction pairs at $E_{1 / 2}=-0.79$ and $-1.25 \mathrm{~V}$ vs $\mathrm{Ag} / \mathrm{AgNO}_{3}$, coupled with the $\mathrm{Co}(\mathrm{II}) / \mathrm{Co}(\mathrm{I})$ pair at $-0.79 \mathrm{~V}$

spectroscopy (Supplementary Fig. 1), electrochemically 40,41 (Fig. 2) and confirmed by bond-valence sums ${ }^{42}$ (Supplementary Table 1). There is one terephthalate strut on a general position and one that lies on an inversion centre. The NDI-py molecule sits on a twofold axis perpendicular to the plane of the molecule. An $\mathrm{N}, \mathrm{N}$-dimethylformamide is coordinated to $\mathrm{Co}(1)$, and a small amount of electron density was modelled as a solvent water at $1 / 4$ occupancy. The diimide was modelled as disordered over two positions related by a small movement along the twofold axis enabled by a slight distortion in coordination of the pyridine to $\mathrm{Co}(1)$. The occupancy of the two components was fixed at 50:50, owing to the presence of the twofold axis. Planarity restraints were applied to some of the rings with additional restraints and constraints to ensure the expected geometry. The coordinated $N$, $N$-dimethylformamide molecule also showed severe thermal motion, and restraints were used to give it and the disordered components reasonable bond lengths, angles and thermal parameters. The large free volume in the unit cell is probably responsible for the uninhibited thermal motion of the struts of the framework, even at $100 \mathrm{~K}$.

Further investigation into these solvent accessible voids gave comparable results of $2643.4 \AA^{3}$ (33.0\% of cell volume) using Olex $2^{43}$ and $2557.6 \AA^{3}$ (31.9\% of cell volume) using Platon ${ }^{44}$, which is high, but on par with similar MOFs, and accounts for the extra solvent content seen by thermogravimetric analysis (Supplementary Fig. 2) $^{13,45}$.

Other $\mathrm{Co}(\mathrm{II})$ coordination polymers with carboxylate and $\mathrm{N}$ donor ligands also contain the same trinuclear building unit of distorted octahedra, as in MOF-CoNDI-py-2 ${ }^{46-51}$. Other MOFs with NDI-py as a ligand display rather different structures. For example, using $\mathrm{Zn}(\mathrm{II})$ as the metal centre, these frameworks usually contain a carboxylate-bridged paddle-wheel building unit, where the metal ions have a tetrahedral or a bidimensional square environment ${ }^{45}$. Previously reported MOFs using Co(II) with NDI-py ligands adopt linear coordinated polymeric structures significantly different from the structure reported here ${ }^{52,53}$.

The simulated powder X-ray diffraction pattern closely matches the experimental data set, as shown in Fig. 3, even with a significant temperature difference (simulated at $100 \mathrm{~K}$ and experimental at $\mathrm{ca} 300 \mathrm{~K}$ ). The main difference between simulated and experimental data are the (200) and (111) peak intensities, assigned to unaccounted solvent molecules in the

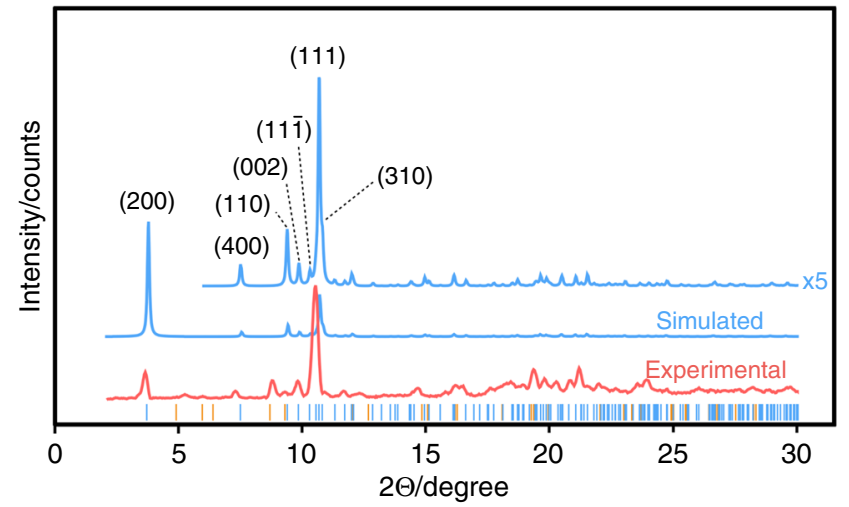

Fig. 3 Experimental and simulated powder $\mathrm{X}$-ray diffractograms of MOFCoNDI-py-2 using a Cu source at $\lambda=1.54 \AA$. Below the curves are the calculated allowed peak positions as black vertical lines

modelled crystal structure and to the flat morphology of the crystals, giving strong preferred orientation effects.

MOF-CoNDI-py-2 has a broad and complicated absorption spectrum, as shown in Fig. 4. Its crystals absorb the whole radiation spectrum between 300 and $2350 \mathrm{~nm}$ except for two wavelengths at 800 and $2075 \mathrm{~nm}$, and this fact is attributed to the low symmetry of the metallic centres. The trinuclear fragment depicted in Fig. 5 has a $\mathrm{C}_{i}$ symmetry, and there are two coordination environments for $\mathrm{Co}(\mathrm{II})$ ions. The first, in the middle, contains a metal ion in a quasi-octahedral site, while the others are equivalent and sit in a $\mathrm{C}_{1}$ symmetry. The resulting spectrum is composed of NDI-py's $\mathrm{S}_{0}-\mathrm{S}_{1}$ transitions around $300-400 \mathrm{~nm}$, a charge transfer band centred at $485 \mathrm{~nm}$, and many weak $d-d$ bands. Nine of these transitions are expected due to the previously mentioned low symmetry of $\mathrm{Co}$ (II) atoms, leading to completely non-degenerate Russell-Saunders terms. In comparison with simple $\mathrm{O}_{h}$ ligand fields, the triply degenerate states$\mathrm{T}_{1 g}$ and $\mathrm{T}_{2 g}$-will split in $3 \mathrm{~A}_{g}$ terms in a $\mathrm{C}_{i}$ coordination symmetry. The observed bands have estimated absorptivities of $10^{3} \mathrm{~L} \mathrm{~mol}^{-1} \mathrm{~cm}^{-1}$, one order of magnitude higher than expected. These observations are probably due to the lower metal symmetry and to metal-ligand $\pi$-bonding ${ }^{54}$, as all coordinated atoms from ligands are $s p^{2}$ in nature. The most intense band in the Vis-NIR spectrum is assigned to a metal-to-ligand charge transfer (MLCT) as the NDI-py ligand has a strong $\pi$-acceptor character, demonstrated by its known reduced species in low electrochemical potentials 34,55 , at $-0.83 \mathrm{~V}$ vs $\mathrm{Fc} / \mathrm{Fc}^{+}$(ferrocene/ferrocinium). Solid-state cyclic voltammetry, shown in Fig. 2, revealed two redox pairs at -0.79 and $-1.25 \mathrm{~V}$ vs $\mathrm{Ag} / \mathrm{AgNO}_{3}$, a characteristic of naphthalene diimides ${ }^{33}, 55$, and this allowed us to estimate the LUMO energy of $-3.9 \mathrm{eV}$, suggesting it is mainly NDI-py in character (see Supplementary Fig. 3).

Photoelectrical characterisation of MOF-CoNDI-py-2. We fabricated the device shown in Fig. 6 for the photoelectrical characterisation of MOF-CoNDI-py-2. The material was analysed by probing every pair of pads, identified as $\mathbf{t} \mathbf{1}, \mathbf{t} \mathbf{2}, \mathbf{b} \mathbf{1}$ and $\mathbf{b} \mathbf{2}$, by applying a sweeping voltage bias under monochromatic light. We studied the material under strong absorption bands (350, 380, $460,510,550$ and $630 \mathrm{~nm}$ ) and weaker bands (700 and $800 \mathrm{~nm})$ in the reflectance spectrum of the framework, as noted in Fig. 4. The crystal face parallel to the substrate, where all four electrodes are connected, is the (100) plane, which is orthogonal to the Co $\cdots$ NDI-py bond and parallel to the Co ${ }^{\cdots}$ TpA bonding plane (see Supplementary Fig. 4). The resulting current vs voltage 


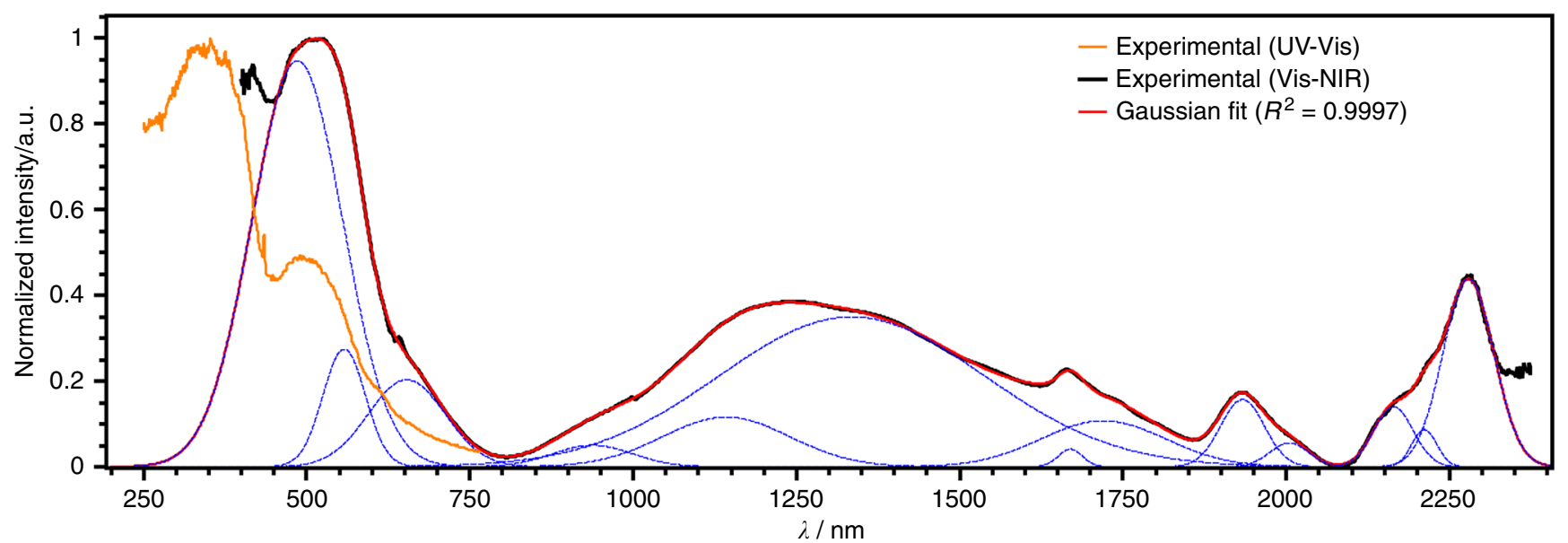

Fig. 4 Comparison of electronic absorption spectra of MOF-CoNDI-py-2. The spectra were collected in the UV-Vis (orange) and Vis-NIR (black) range. Mathematically deconvoluted Gaussians for the Vis-NIR, only, are shown in blue dashed lines. The sum of all individual Gaussians is shown in red

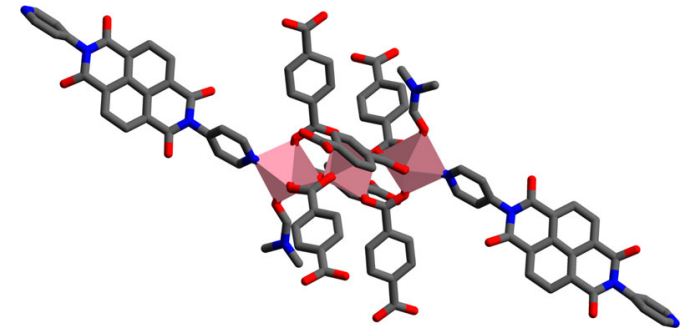

Fig. 5 Spectroscopic unity of MOF-CoNDI-py-2. It is composed of three metal ions, six TpA anions, two NDI-py and two DMF molecules. Hydrogen atoms are not shown for clarity

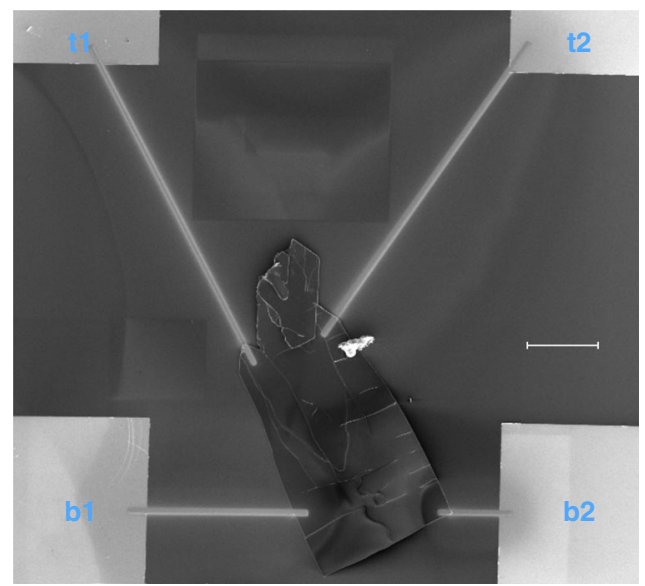

Fig. 6 SEM image of the device used in all electrical and photoelectrical characterisations. It consists of a $\mathrm{p}-\mathrm{Si} / \mathrm{SiO}_{2}$ wafer onto which a MOFCoNDI-py-2 single crystal is connected to Au pads, labelled t1, b1, t2 and $\mathbf{b 2}$, by deposited Pt electrodes. Scale bar $=100 \mu \mathrm{m}$

curves are highly anisotropic and wavelength-dependent. The whole set can be found in the Supplementary Figs. 5-8. Here we show in Fig. 7 the responsivity measurements of all probed directions when excited with a $510 \mathrm{~nm}$ wavelength. In this case, this wavelength is mainly charge-transfer in character, with a low contribution of $d-d$ transition. The highly asymmetric nature is indicative of a metal-semiconductor contact, or Schottky, effect. The metal-ligand interactions forms nanoscale Schottky diodes

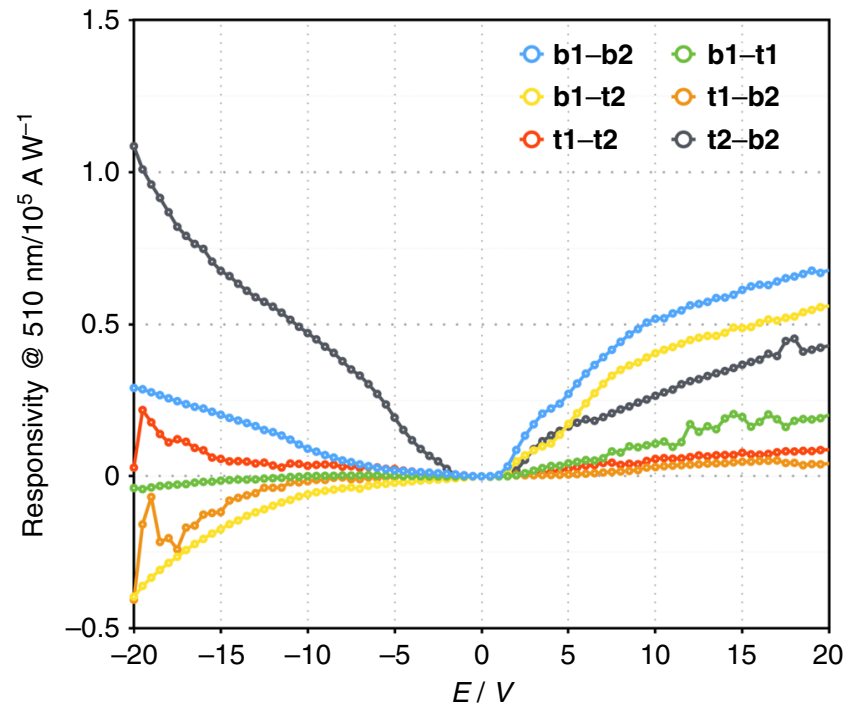

Fig. $7 \mathrm{MOF}$ responsivity under monochromatic light illumination.

Responsivity measurements of the MOF device probing all directions under a $510 \mathrm{~nm}$ monochromatic light

throughout the crystal, providing a high built-in field that efficiently dissociates the excitons generated under illumination, and this is further supported by the very high photocurrents observed discussed below.

\section{Discussion}

The crystal displays photoresponse intensities (Jph) proportional to band intensities at that wavelength, as shown in Fig. 8, at which the maximum outputs coincide with the MLCT band. We believe that the conduction mechanism involves a charge transfer from the metal centre to the strong $\pi$-acceptor NDI-py, promoting hole transport through the Co $\cdots \mathrm{TpA}$ direction while electrons are transported by the NDI-py direction. While the distance between NDI-py unities are unusually high for charge transport, these are not far off from similar structures used as high-mobility active materials in transistors (up to $8.3 \AA)^{30}$. Excitation at the MLCT band further improves this mechanism by promoting a charge injection from metal to diimide. All orientations follow the same trend however directions involving t1 display a sharp Jph 


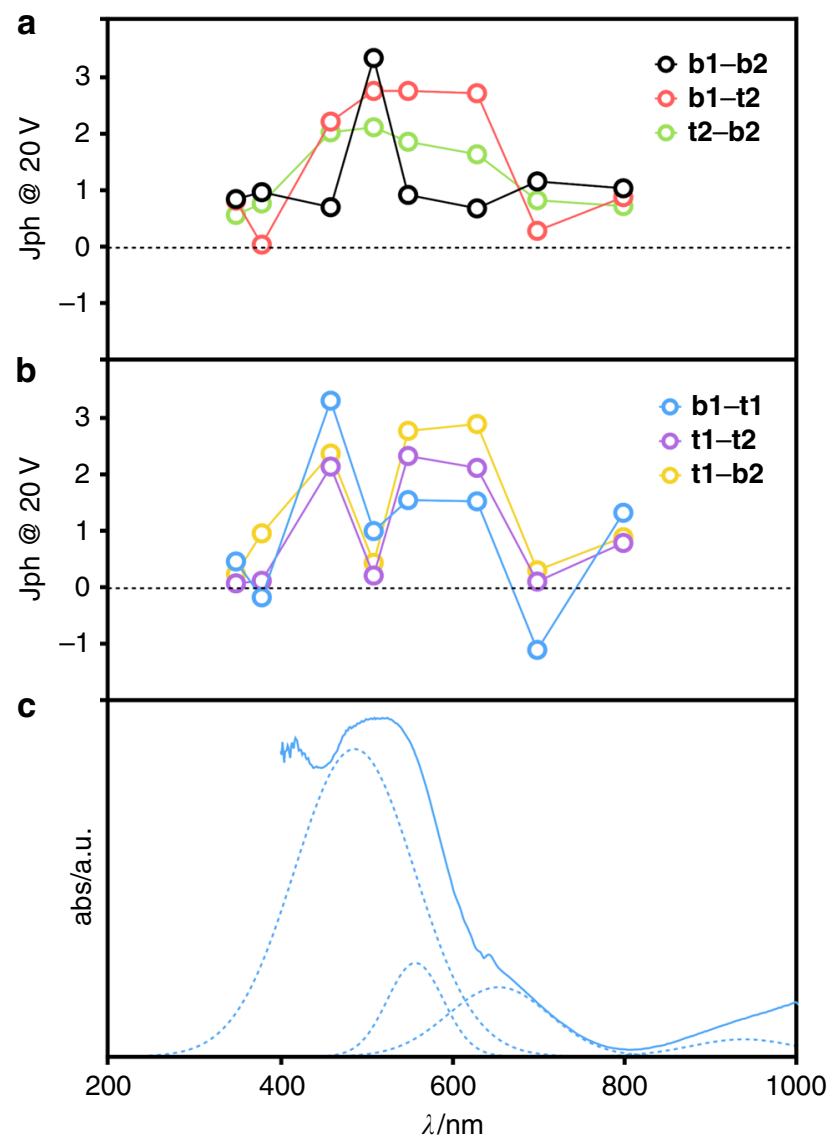

Fig. 8 Comparison of photoresponse intensities at $20 \mathrm{~V}$ with the deconvoluted absorption spectrum of MOF-CoNDI-py-2. a, b Photoresponse intensities at $20 \mathrm{~V}$. c Electronic absorpotion spectrum of MOF-CoNDI-py-2

decrease at $510 \mathrm{~nm}$, and this is attributed to the fact that $\mathbf{t} \mathbf{1}$ sits on a different crystal layer than all the other pads. This introduces a significant $z$-axis component, orthogonal to the (100) crystal face and through the Co $\cdots$ NDI-py bond, to the electron path not experienced in this degree by any other pad, and this additional crystal direction in all combinations involving $\mathbf{t} 1$ leads to a slightly distorted behaviour. Responsivities at $20 \mathrm{~V}$ were at very high values of $2.5 \times 10^{5} \mathrm{~A} \mathrm{~W}^{-1}(\mathbf{t} 2-\mathbf{b} 2$ at $800 \mathrm{~nm})$, and followed the same trend as Jph although they were unexpectedly higher at 800 $\mathrm{nm}$, which indicates the crystal's capacity to harvest photons efficiently even at wavelengths with low absorptivity. In Supplementary Table 2, we compare the observed responsivity of MOFCoNDI-py-2 to other materials. Measurements of the photocurrent under different illumination intensities $\left(20-100 \mathrm{~mW} \mathrm{~cm}^{-2}\right.$, see Supplementary Figs. 9 and 10) indicate a linear response under an applied bias of $\pm 20 \mathrm{~V}$, which equates to a bulk electric field of less than $0.1 \mathrm{~V} \mathrm{\mu m}^{-1}$. The observation of a linear behaviour over such a wide illumination intensity indicates no variation in both the external as well as internal quantum efficiencies for the range of illumination intensities studied. Furthermore, the linear photoresponse under such high illumination intensities also eliminates thermal effects as the origin for the high photoresponses observed ${ }^{56}$. We believe part of the reason for these very high responsivities are the high surface areas, each with an electric field across the device. As a result the depleted active region can act upon the total optical flux, giving high responsivity with large sensory volumes. The high responsivities observed here are indicative of an efficient exciton dissociation mechanism.
Efficient exciton dissociation requires the presence of strong built-in fields, which far exceed the bulk fields applied to the device here. Such strong nanoscale fields are expected due to the interaction between the metal ion and the organic ligand. We note that there is evidence of high photoactivity in highly crystalline $\mathrm{MOFs}^{57}$. While exciton dissociation as a result of a Schottky contact at the metal/MOF interface is also possible, this is considered to be highly unlikely as the shading effect by the metal reduces the photocurrent generation. Except for the t1-b1 direction, the device displayed positive Jph and responsivities at positive bias while at negative bias, with the exception of the $\mathbf{t} \mathbf{2}-\mathbf{b} \mathbf{2}$ direction, the device had a dual photoconductive-photoresistive behaviour depending on the incident wavelength.

Current vs voltage measurements carried out in darkness indicate asymmetric characteristics for the MOF crystal with $\mathrm{Pt}$ contacts. This is ascribed to the anisotropic charge semiconduction and different charge transport properties along the Co $\cdots$ NDI-py direction as opposed to the direction perpendicular to aromatic rings. The electrical characteristics are expected to be further influenced by the different electronic properties at the crystal faces at which the Pt metal comes into contact with the MOF crystal ${ }^{58-60}$.

Observation of the photocurrent characteristics under negative bias for monochromatic illumination reveals a mixture of positive and negative photocurrents, as opposed to the positive only photocurrent values expected. This is in contrast to the photoresponse under white light bias measurements where there does not appear to be a mix of positive and negative responses. It is noted here that there is a significant difference in the illumination intensities used under monochromatic response $\left(\mathrm{nW} \mathrm{cm}^{-2}\right)$ measurements as opposed to white light measurements $\left(\mathrm{mW} \mathrm{cm}^{-2}\right)$. As a result, the dark current density of the material under different configurations plays a significant role in the sign of the photoresponse observed, especially at lower intensities as used in observing the monochromatic response. Light intensities at which the photocurrent generated is lower than the dark current density results in a negative photocurrent response. This effect is further amplified by the anisotropic semiconducting properties of the crystal, the optical absorption coefficients under different wavelengths, as well the properties of the interfacial contacts between the metal and the crystal faces. However, these factors are less likely to affect the photocurrent response under higher light intensities as those used for the measurements carried out under white light due to the significantly higher photocurrents generated.

An interesting feature of the MOF crystals synthesised in this work is the observation of both photo-conductance (increase in the current under illumination) and inverse photo-conductance (or photo resistance, reduction of current under illumination). We note that previously, Nakanishi et al. have reported both photo and inverse photo-conductance characteristics which was ascribed to the combination of a self-assembled monolayer and the metal nanoparticle used ${ }^{61}$. One possible reason for the observed inverse photo-semiconduction in this work is the ability of the metal centres to act as charge trap sites due to their oxidation state. This follows from the expected energy diagram formed upon complexation, as the HOMO level will have a major contribution from the ligands, thus being more localised in the organic components while the SOMO will have a large Co character $^{39}$. This energy alignment favours hole trapping in Co (II) ions. However interestingly, the crystal does not appear to indicate inverse photo-semiconductivity for the same group of wavelengths probed for the different combinations of electrical contacts, indicating that both the bulk and the contact properties are likely to play an important role in the observed photo and inverse photo-semiconduction characteristics. 
Despite its anisotropy that lead to different conductivity values, the crystal conducts electricity in all the directions that could be measured by our four-point probe apparatus, showing the whole structure is conductive, and that electrons would not be transferred between the metallic centres exclusively by NDI moieties as one could expect, due to its semiconductive properties and conjugation that favours charge delocalisation. The results suggest that long-distance electron transfer and terephthalate-mediated electron transfer may happen as well.

In summary, we have synthesised and fully characterised MOF-CoNDI-py-2, a naphthalene diimide-based metal-organic framework featuring high crystallinity, electrochemical activity, electrical semiconductivity and photoactivity. The observed anisotropic electrical semiconduction also features a photoresistive-photoresponsive dual behaviour and one of the highest responsivities ever reported, which opens a new horizon in the development of MOF devices.

\section{Methods}

Synthesis of NDI-py. The synthesis of N,N-bis(4-pyridyl)-1,4,5,8-naphthalene diimide is described in the literature ${ }^{62}$, where we followed an adapted route ${ }^{63}$, using $503.7 \mathrm{mg}(1.88 \mathrm{mmol})$ of 1,4,5,8-naphthalic dianhydride (Aldrich), $543.4 \mathrm{mg}$ $(5.77 \mathrm{mmol}$ ) of 4 -aminopyridine (Acros) in ca $2 \mathrm{~g}$ of molten imidazole (Vetec), heated in an oil bath for $30 \mathrm{~min}$ and allowed to cool naturally to room temperature. The resulting solid was treated with a mixture of $\mathrm{H}_{2} \mathrm{O}: \mathrm{EtOH}: \mathrm{HNO}_{3}$ 10:10:1 (v/v, Synth) and filtered, washed again with the same solution and then sequentially with $\mathrm{H}_{2} \mathrm{O}$, EtOH (Synth) and $\mathrm{Et}_{2} \mathrm{O}$ (Synth). The resulting product was a beige solid with $94 \%$ yield, $742.1 \mathrm{mg}$. This solid was then recrystallised in DMF with $47 \%$ yield. ${ }^{1} \mathrm{H}-$ NMR ( $\left.\mathrm{CF}_{3} \mathrm{COOD}, \mathrm{ppm}\right): 9.11(4 \mathrm{H}, \mathrm{d}, J=6.5 \mathrm{~Hz}) ; 9.03(4 \mathrm{H}, \mathrm{s}) ; 8.37(4 \mathrm{H}, \mathrm{d}, J=6.5$ $\mathrm{Hz}) .{ }^{13} \mathrm{C}-\mathrm{NMR}\left(\mathrm{CF}_{3} \mathrm{COOD}, \mathrm{ppm}\right): 164.8 ; 155.0 ; 145.1 ; 135.0 ; 131.4 ; 129.7 ; 128.8$. FTIR $\left(\mathrm{KBr}, \mathrm{cm}^{-1}\right): 3065,3035,1716,1674,1590,1580,1501,1491,1448,1414$, $1350,1251,1214,1198,1148,1123,985,863,827,767,751,718,623,529$. MS $(\mathrm{m} /$ $z): 421[\mathrm{M}+\mathrm{H}]^{+}, 211[\mathrm{M}+2 \mathrm{H}]^{2+}$. See Supplementary Figs. 3 and $11-16$ and Supplementary Note 1 for reaction scheme, NMR, UV-Vis, FTIR and electrochemical data.

Synthesis of MOF-CoNDI-py-2. The metal-organic framework was synthesised by a solvothermal route, dissolving $0.24 \mathrm{mmol}$ of NDI-py, $0.48 \mathrm{mmol}$ of TpA and $0.48 \mathrm{mmol}$ of $\mathrm{Co}\left(\mathrm{NO}_{3}\right)_{2} \cdot 6 \mathrm{H}_{2} \mathrm{O}$ in $50 \mathrm{~mL}$ of $\mathrm{N}, \mathrm{N}$-dimethylformamide. This solution was then transferred to a polytetrafluoroethylene vial within a stainless steel casing. The vessel was heated at $80^{\circ} \mathrm{C}$ for $48 \mathrm{~h}$ and allowed to cool to room temperature. Crystals were collected by filtration and washed thoroughly with $\mathrm{N}, \mathrm{N}$-dimethylformamide. Yield, based on NDI-py ligand, was 50\%. See Supplementary Tables 2 and 3 for bond-valence sums and crystal data, and Supplementary Figs. 1, 2 and 16 for EPR, TGA and FTIR data.

X-ray diffraction. Powder samples were dried in an oven at $300{ }^{\circ} \mathrm{C}$ for $3 \mathrm{~h}$ and analysed in a Siemens D5005 diffractometer with a $\mathrm{Cu}$ wavelength, using $\mathrm{K} \alpha$, at $0.02^{\circ} \mathrm{C} \mathrm{min}-1$. For the single crystal analysis, a suitable crystal of MOF-CoNDI-py2 was selected and mounted on a Mitegen loop with Fromblin oil and placed on a Bruker-Nonius APEX II CCD diffractometer equipped with a Bruker-Nonius FR591 rotating anode at the UK's National Crystallography Service, University of Southampton ${ }^{64}$. The crystal was kept at $100(2) \mathrm{K}$ during data collection. Using Olex $2^{43}$, the structure was solved with the ShelXS structure solution programme using Direct Methods and refined with the ShelXL refinement package using Least Squares minimisation ${ }^{65}$. CCDC 1517923 contains the supplementary crystallographic data for this paper. These data can be obtained free of charge from the Cambridge Crystallographic Data Centre.

Crystal face indexing. A suitable crystal was selected and mounted on a glass fibre with Fromblin oil, as shown in Supplementary Fig. 17, and placed on a Rigaku Oxford Diffraction SuperNova diffractometer with a dual source ( $\mathrm{Cu}$ at zero) equipped with an AtlasS2 CCD area detector. The crystal was kept at 100(2) K during data collection. Data were recorded with $\mathrm{CuK}_{\alpha}$ radiation and frames, data processing and indexing was performed with the CrysAlisPro 1.171.38.43f software (Rigaku Oxford Diffraction, 2015).

Simulated powder X-ray diffraction. The X-ray powder pattern was calculated using CCDC's (Cambridge Crystallographic Data Centre, University of Cambridge, UK) Mercury software, using a Cu source $(\lambda=1.54056 \AA), 0.02$ degree step and 0.1 units of $2 \Theta$ of full-width at half-maximum.
EPR spectroscopy. X-band EPR spectra were recorded at 7 and at $25 \mathrm{~K}$ with a EMX Bruker spectrometer equipped with a ER4116DM Bruker cavity, an Oxford Instrument Cryostat (ESR900) and a Bruker temperature controller (ER4131VT).

Electronic absorption spectra. Electronic absorption spectra of MOF-CoNDI-py2 crystals were collected by diffusive reflectance spectroscopy using an Analytical Spectral Devices FieldSpec 3 spectrophotometer for the Vis-NIR region (350-2500 $\mathrm{nm}$ ) and an Ocean Optics DH-2000-Ball for the UV-Vis region (250-800 nm), both using $\mathrm{MgO}$ (Synth) as reference.

Fourier transform infrared spectra. FTIR spectra of NDI-py, TpA and MOFCoNDI-py-2 were acquired in a Shimadzu IRPrestige-21 Fourier transform infrared spectrophotometer using $\mathrm{KBr}$ pellets.

Polarised Raman spectra. Data were acquired using a Witec Alpha $300 \mathrm{R}$ with a $532 \mathrm{~nm} 7.5 \mathrm{~mW} \mathrm{~cm}^{-2} \mathrm{Nd}$ :YAG laser. Polarisers at the source and detector were positioned and rotated relative to one another at the indicated angles. Crystals of MOF-CoNDI-py-2 were deposited on a glass slide from a DMF suspension and dried on a hot plate. See Supplementary Figs. 18 and 19, and Supplementary Note 2 for spectra and discussion.

Electrochemistry. Cyclic voltammetry of NDI-py was recorded with an Autolab PGSTAT30 Potentiostat/Galvanostat at $25 \mathrm{mV} \mathrm{s}^{-1}$ using a $1 \mathrm{mmol} \mathrm{dm}^{-3}$ solution in DMF (Aldrich) containing $10 \mathrm{mmol} \mathrm{dm}{ }^{-3}$ of $\left[\mathrm{N}(n-\mathrm{Bu})_{4}\right]\left(\mathrm{PF}_{6}\right)$ (Aldrich) as supporting electrolyte, a glassy carbon electrode $(\phi=1 \mathrm{~mm})$ as working electrode, a $1 \mathrm{~cm}^{2} \mathrm{Pt}$ counter electrode, a silver wire as pseudo-reference electrode and ferrocene (Aldrich) as internal reference.

Solid-state electrochemistry. Cyclic voltammetry of MOF-CoNDI-py-2 was recorded at $50 \mathrm{mV} \mathrm{s}^{-1}$ in a EG\&G model 173 Potentiostat/Galvanostat equipped with a PAR model universal programmer and a PAR model 179 digital coulometer using a cavity microelectrode $\left(\phi=50 \mu \mathrm{m}, d=25 \mu \mathrm{m}\right.$ and $\left.V=1 \times 10^{-8} \mathrm{~cm}^{3}\right)$ in a three-electrode arrangement, with an $\mathrm{Ag}$ wire as counter electrode, $\mathrm{Ag} / \mathrm{AgNO}_{3}$ (Aldrich, $1 \times 10^{-2} \mathrm{~mol} \mathrm{dm}^{-3}$ in $\mathrm{MeCN}$ ) as reference electrode and $0.1 \mathrm{~mol} \mathrm{dm}^{-3}$ $\left[\mathrm{N}(n-\mathrm{Bu})_{4}\right]\left(\mathrm{PF}_{6}\right)($ Aldrich) in MeCN (Aldrich) as supporting electrolyte.

Electron microscopy. Scanning electron microscopy images of the fabricated devices were taken using a FEI Nova Nanolab 600.

Electronic device. A $200 \mathrm{~nm} \mathrm{SiO}$ layer was deposited onto a $p$-Si substrate via plasma-enhanced chemical vapour deposition (PECVD). Gold electrodes were deposited onto this $\mathrm{Si} / \mathrm{SiO}_{2}$ substrate by lithography, with a pre-layer of titanium for adherence. These were cleaned with ultrasound bath in acetone for $5 \mathrm{~min}$ and dried over nitrogen flow. MOF-CoNDI-py-2 crystals were then deposited from acetone suspensions and connected to the Au electrodes by Pt electrodes, via vapour deposition using the sublimating compound trimethyl(methylcyclopentadienyl)platinum(IV) (Aldrich, 98\%) as source. A crucible containing this compound, within the Focused Ion Beam (FIB) system in a FEI Nova Nanolab 600 dua beam system, is slowly heated to $50^{\circ} \mathrm{C}$ and the vapour is directed to the desired location through a needle.

Electrical characterisation. Current vs voltage curves of the fabricated devices were determined in air using two spring loaded probes using a Keithley 2400 in a two-point probe arrangement. Photoresponses were measured using a Bentham PVE300 Quantum Efficiency with the same devices.

Photoconductivity measurements. The photoconductivity measurements were carried out using a Bentham PVE300 system consisting of xenon quartz halogen lamps. The appropriate wavelength was selected using the built-in monochromator in the system. The device was placed inside a dark box, which consists of ports connected to the lamps. The devices were connected to a Keithely 2400 operated in a four-wire configuration and two spring loaded probes were used to contact the lithographically patterned contact pads. The temperature of the device was maintained at $25 \pm 0.5^{\circ} \mathrm{C}$ using a built-in temperature controlling stage in the setup. The output intensities of the two lamps are given in Supplementary Table 4. The responsivity was calculated by using Eq. (1).

$$
\text { Responsivity }=\frac{I_{i t}-I_{d}}{W \times\left(\frac{A_{c}}{A_{s}}\right)}
$$

In the equation above, $I_{\mathrm{it}}$ is the total current under illumination, $I_{\mathrm{d}}$ is the dark current, $W$ is the illumination intensity, $A_{c}$ is the crystal area and $A_{\mathrm{s}}$ is the area of the spot size where the crystal is placed. In order to minimise errors in estimating the crystal area, the SEM micrograph was used to calculate the crystal area. 
Thermogravimetric analysis. TGA and DTG analysis were carried out in a TA Instruments SDT 2960 Simultaneous DTA-TGA Thermal Analyst 2100 in oxidising atmosphere from room temperature to $1000^{\circ} \mathrm{C}$ at $10^{\circ} \mathrm{C} \mathrm{min}-1$

Data availability. CCDC 1517923 contains the crystallographic data for MOFCoNDI-py-2. The data can be obtained free of charge from the Cambridge Crystallographic Data Centre via www.ccdc.cam.ac.uk/getstructures. All the other data are available from the authors on reasonable request.

Received: 5 December 2016 Accepted: 14 November 2017

Published online: 15 December 2017

\section{References}

1. Batten, S. R. et al. Terminology of metal-organic frameworks and coordination polymers (IUPAC Recommendations 2013). Pure. Appl. Chem. 85, 1715-1724 (2013).

2. Kitagawa, S., Kitaura, R. \& Noro, S. Functional porous coordination polymers. Angew. Chem. Int. Ed. 43, 2334-2375 (2004).

3. Furukawa, H., Cordova, K. E., O’Keeffe, M. \& Yaghi, O. M. The chemistry and applications of metal-organic frameworks. Science 341, 6149 (2013).

4. Wriedt, M. et al. Low-energy selective capture of carbon dioxide by a predesigned low-energy selective capture of carbon dioxide by a pre-designed elastic single-molecule trap. Angew. Chem. Int. Ed. 51, 1-6 (2012).

5. Wang, C., Wang, J.-L. \& Lin, W. Elucidating molecular iridium water oxidation catalysts using metal organic frameworks: a comprehensive structural, catalytic, spectroscopic, and kinetic study. J. Am. Chem. Soc. 134, 19895-19908 (2012).

6. Allendorf, M. D. et al. Guest-induced emergent properties in metal organic frameworks. J. Phys. Chem. Lett. 6, 1182-1195 (2015).

7. Allendorf, M. D., Medishetty, R. \& Fischer, R. A. Guest molecules as a design element for metal-organic frameworks. MRS Bull. 41, 865-869 (2016).

8. Sun, L., Miyakai, T., Seki, S. \& Dincă, M. $\mathrm{Mn}_{2}$ (2,5-disulfhydrylbenzene-1,4dicarboxylate): a microporous metal organic framework with infinite $(\mathrm{MnS})$ chains and high intrinsic charge mobility. J. Am. Chem. Soc. 135, 8185-8188 (2013).

9. Sun, L., Hendon, C. H., Minier, M. A., Walsh, A. \& Dincă, M. Million-fold electrical conductivity enhancement in $\mathrm{Fe}_{2}(\mathrm{DEBDC})$ versus $\mathrm{Mn}_{2}(\mathrm{DEBDC})(\mathrm{E}=$ S, O). J. Am. Chem. Soc. 137, 6164-6167 (2015)

10. Park, S. S. et al. Cation-dependent intrinsic electrical conductivity in isostructural tetrathiafulvalene-based microporous metal organic frameworks. J. Am. Chem. Soc. 137, 1774-1777 (2015).

11. Wade, C. R., Li, M. \& Dincă, M. Facile deposition of multicolored electrochromic metal-organic framework thin films. Angew. Chem. Int. Ed. 125, 1-6 (2013).

12. Sheberla, D. et al. Conductive MOF electrodes for stable supercapacitors with high areal capacitance. Nat. Mater. 16, 220-224 (2016).

13. Ma, B.-Q., Mulfort, K. L. \& Hupp, J. T. Microporous pillared paddle-wheel frameworks based on mixed-ligand coordination of zinc ions. Inorg. Chem. 44, 4912-4914 (2005).

14. Mulfort, K. L. \& Hupp, J. T. Chemical reduction of metal-organic framework materials as a method to enhance gas uptake and binding. J. Am. Chem. Soc. 129, 9604-9605 (2007).

15. Zhang, Z. \& Awaga, K. Redox-active metal-organic frameworks as electrode materials for batteries. MRS Bull. 41, 883-889 (2016).

16. D'Alessandro, D. M. Exploiting redox activity in metal-organic frameworks: concepts, trends and perspectives. Chem. Commun. 52, 8957-8971 (2016).

17. Felblyum, J. I., Keenan, E. A., Matzger, A. J. \& Maldonado, S. Photoresponse characteristics of archetypal metal-organic frameworks. J. Phys. Chem. C 116, 3112-3121 (2012).

18. Dolgopolova, E. A. \& Shustova, N. B. Metal-organic framework photophysics: Optoelectronic devices, photoswitches, sensors, and photocatalysts. MRS Bull. 41, 890-896 (2016).

19. Sun, L., Campbell, M. G. \& Dincă, M. Electrically conductive porous metal-organic frameworks. Angew. Chem. Int. Ed. 55, 3566-3579 (2016).

20. Leong, C. F., Usov, P. M. \& D’Alessandro, D. M. Intrinsically conducting metal-organic frameworks. MRS Bull. 41, 858-864 (2016).

21. Walsh, A., Butler, K. T. \& Hendon, C. H. Chemical principles for electroactive metal-organic frameworks. MRS Bull. 41, 870-876 (2016).

22. Kambe, T. et al. $\pi$-conjugated nickel bis(dithiolene) complex nanosheet. J. Am. Chem. Soc. 135, 2462-2465 (2013)

23. Kambe, T. et al. Redox control and high conductivity of nickel Bis(dithiolene) complex $\pi$-nanosheet: a potential organic two-dimensional topological insulator. J. Am. Chem. Soc. 136, 14357-14360 (2014).

24. Pal, T. et al. Interfacial synthesis of electrically conducting palladium Bis (dithiolene) complex nanosheet. ChemPlusChem. 80, 1255-1258 (2015).

25. Sakamoto, R. et al. A photofunctional bottom-up bis(dipyrrinato)zinc(II) complex nanosheet. Nat. Commun. 6, 6713 (2015).
26. Sakamoto, R. et al. The coordination nanosheet (CONASH). Coord. Chem. Rev 320-321, 118-128 (2016).

27. Vinogradov, A. V. et al. The first depleted heterojunction $\mathrm{TiO}_{2} / \mathrm{MOF}$-based solar cell. Chem. Commun. 50, 10210-10213 (2014).

28. Lee, D. Y. et al. Enhanced photovoltaic performance of $\mathrm{Cu}$-based metal-organic frameworks sensitized solar cell by addition of carbon nanotubes. Sci. Rep. 4, 1-5 (2014).

29. Dincă, M. \& Léonard, F. Metal-organic frameworks for electronics and photonics. MRS Bull. 41, 854-857 (2016).

30. Shukla, D. et al. Thin-film morphology control in naphthalene-diimide-based semiconductors: high mobility n-type semiconductor for organic thin-film transistors. Chem. Mater. 20, 7486-7491 (2008).

31. Yuan, Z. et al. Core-fluorinated naphthalene diimides: synthesis, characterization, and application in n-type organic field-effect transistors. $\mathrm{Org}$ Lett. 18, 456-459 (2016).

32. $\mathrm{Wu}, \mathrm{Z}$. et al. $\mathrm{n}$-Type water/alcohol-soluble naphthalene diimide-based conjugated polymers for high-performance polymer solar cells. J. Am. Chem. Soc. 138, 2004-2013 (2016).

33. Rozanski, L. J. et al. Solution processed naphthalene diimide derivative as electron transport layers for enhanced brightness and efficient polymer light emitting diodes. J. Mater. Chem. C 1, 3347-3352 (2013).

34. Bhosale, S. V., Jani, C. H. \& Langford, S. J. Chemistry of naphthale diimides. Chem. Soc. Rev. 37, 331-342 (2008).

35. Xie, Y.-X., Zhao, W.-N., Li, G.-C., Liu, P.-F. \& Han, L. A naphthalenediimidebased metal organic framework and thin film exhibiting photochromic and electrochromic properties. Inorg. Chem. 55, 549-551 (2015).

36. McCarthy, B. D., Hontz, E. R., Yost, S. R., Voorhis, T. V. \& Dincă, M. Charge transfer or J-coupling? Assignment of an unexpected red-shifted absorption band in a naphthalenediimide-based metal-organic framework. J. Phys. Chem. Lett. 4, 453-458 (2013).

37. Usov, P. M., Fabian, C. \& D’Alessandro, D. M. Rapid determination of the optical and redox properties of a metal-organic framework via in situ solid state spectroelectrochemistry. Chem. Commun. 48, 3945-3947 (2012).

38. Ling, Y. et al. Electronic structure of positive and negative polarons in functionalized dithienylthiazolo5,4-dthiazoles: a combined EPR and DFT study. Phys. Chem. Chem. Phys. 16, 10032-10040 (2014).

39. Kettle, S. F. A. Physical Inorganic Chemistry - A Coordination Chemistry Approach 1st edn (Spektrum Academic Publishers, Oxford, 1996).

40. Isse, A. A., Gennaro, A. \& Vianello, E. The electrochemical reduction mechanism of N,N'-i,2-phenylene-bis(salicylideneiminato)cobalt(II). J. Am. Chem. Soc. Dalton Trans. 14, 2091-2096 (1993).

41. Cañadas, M., L.-Torres, E., M.-Arias, A., Mendiola, M. A. \& Sevilla, M. T. Spectroscopic and electrochemical properties of nickel(II), iron(III) and cobalt (II) complexes with benzilbisthiosemicarbazone-importance of working conditions and the metal salt used in the final complex. Polyhedron 19, 2059-2068 (2000).

42. Brese, N. E. \& O'Keeffe, M. Bond-valence parameters for solids. Acta Cryst. B 47, 192-197 (1991).

43. Dolomanov, O. V., Bourhis, L. J., Gildea, R. J., Howard, J. A. K. \& Puschmann, H. OLEX2: a complete structure solution, refinement and analysis program. J. Appl. Crystallogr. 42, 339-341 (2009).

44. Spek, A. L. Structure validation in chemical crystallography. Acta Cryst. D 65 148-155 (2009).

45. Deng, H.-Y., He, J.-R., Pan, M., Li, L. \& Su, C.-Y. Synergistic metal and anion effects on the formation of coordination assemblies from a $n, n^{\prime}$-bis(3-pyridylmethyl) naphthalene diimide ligand. Cryst. Eng. Comm. 11, 909-917 (2009).

46. Poulsen, R. D., Bentien, A., Christensen, M. \& Iversen, B. B. Solvothermal synthesis, multi-temperature crystal structures and physical properties of isostructural coordination polymers, $2 \mathrm{C}_{4} \mathrm{H}_{1} 2 \mathrm{~N}^{+} M_{3}\left(\mathrm{C}_{8} \mathrm{H}_{4} \mathrm{O}_{4}\right)_{4}^{2}-\cdot 3 \mathrm{C}_{5} \mathrm{H}_{1} 1 \mathrm{NO}, M$ = Co, Zn. Acta Cryst. B 62, 245-254 (2006).

47. Clausen, H. F., Overgaard, J., Chen, Y. S. \& Iversen, B. B. Synchrotron X-ray charge density study of coordination polymer $\mathrm{Co}_{3}\left(\mathrm{C}_{8} \mathrm{H}_{4} \mathrm{O}_{4}\right)_{4}\left(\mathrm{C}_{4} \mathrm{H}_{12} \mathrm{~N}\right)_{2}\left(\mathrm{C}_{5} \mathrm{H}_{11} \mathrm{NO}\right)_{3}$ at 16 K. J. Am. Chem. Soc. 130, 7988-7996 (2008).

48. Zhang, J. et al. Urothermal synthesis of crystalline porous materials. Angew. Chem. Int. Ed. 49, 8876-8879 (2010).

49. Wang, X.-F., Zhang, Y.-B., Xue, W., Qi, X.-L. \& Chen, X.-M. Two temperatureinduced isomers of metal-carboxylate frameworks based on different linear trinuclear $\mathrm{Co}_{3}(\mathrm{RCOO})_{8}$ clusters exhibiting different magnetic behaviours. CrystEngComm 12, 3834-3839 (2010).

50. Wang, Y., Zhao, F.-H., Che, Y.-X. \& Zheng, J.-M. A 3D photoluminescent Cd (II) polymer based on mixed 3,5-bis-oxyacetate-benzoic acid and rigid bis (imidazole) ligands with an unusual $(4,8)$-connected topology. Inorg. Chem. Commun. 17, 180-183 (2012).

51. Munn, A. S. et al. Metal organic frameworks from divalent metals and 1,4benzenedicarboxylate with bidentate pyridine-N-oxide Co-ligands. Cryst. Growth Des. 15, 891-899 (2015). 
52. Tao, T. et al. The first observation of one-dimensional naphthalenediimidatobased transition-metal coordination polymers: syntheses, crystal structures and properties. Cryst. Growth Des. 12, 4580-4587 (2012).

53. Fang, X., Yuan, X., Song, Y.-B., Wang, J.-D. \& Lin, M.-J. Cooperative lone pair$\pi$ and coordination interactions in naphthalene diimide coordination networks. CrystEngComm 16, 9090-9095 (2014).

54. Clegg, W., Little, I. R. \& Straughan, B. P. Zinc carboxylate complexes: structural characterization of the mixed-metal linear trinuclear complexes $\mathrm{MZn}_{2}(\mathrm{crot})_{6}$, (base) $)_{2},\left(\mathrm{M}=\mathrm{Mn}, \mathrm{Co}, \mathrm{Ni}, \mathrm{Zn}, \mathrm{Cd}, \mathrm{Mg}, \mathrm{Ca}, \mathrm{Sr} ; \mathrm{crot}^{-}=\right.$Crotonate $(1-)$; Base $=$ Quinoline, 6-Methylquinoline). Inorg. Chem. 27, 1916-1923 (1988).

55. Castaldelli, E., Triboni, E. R. \& Demets, G. J.-F. Self-assembled naphthalenediimide derivative films for light-assisted electrochemical reduction of oxygen. Chem. Commun. 47, 5581-5583 (2011).

56. Xu, X., Gabor, N. M., Alden, J. S., van der Zande, A. M. \& McEuen, P. L. Photothermoelectric effect at a graphene interface junction. Nano Lett. 10, 562-566 (2010).

57. Liu, J. et al. Photoinduced charge-carrier generation in epitaxial MOF thin films: high efficiency as a result of an indirect electronic band gap? Angew. Chem. Int. Ed. 54, 1-6 (2015)

58. Kuo, C.-H., Yang, Y.-C., Gwo, S. \& Huang, M. H. Facet-dependent and au nanocrystal-enhanced electrical and photocatalytic properties of $A u-\mathrm{Cu}_{2} \mathrm{O}$ core-shell heterostructures. J. Am. Chem. Soc. 133, 1052-1057 (2011).

59. Kim, C. W., Yeob, S. J., Cheng, H.-M. \& Kang, Y. S. A selectively exposed crystal facet-engineered $\mathrm{TiO}_{2}$ thin film photoanode for the higher performance of the photoelectrochemical water splitting reaction. Energy Environ. Sci. 8, 3646-3653 (2015).

60. Tan, C.-S., Hsu, S.-C., Ke, W.-H., Chen, L.-J. \& Huang, M. H. Facet-dependent electrical conductivity properties of $\mathrm{Cu}_{2} \mathrm{O}$ crystals. Nano Lett. 15, 2155-2160 (2015).

61. Nakanish, H. et al. Photoconductance and inverse photoconductance in films of functionalized metal nanoparticles. Nature 460, 371-375 (2009).

62. Dinolfo, P. H., Williams, M. E., Stern, C. L. \& Hupp, J. T. Rhenium-based molecular rectangles as frameworks for ligand-centered mixed valency and optical electron transfer. J. Am. Chem. Soc. 126, 12989-13001 (2004).

63. Marcon, R. O. \& Brochsztain, S. Characterization of self-assembled thin films of zirconium phosphonate/aromatic diimides. Thin Solid Films 492, 30-34 (2005).

64. Coles, S. J. \& Gale, P. A. Changing and challenging times for service crystallography. Chem. Sci. 3, 683-689 (2012).

65. Sheldrick, G. M. A short history of SHELX. Acta Cryst. A 64, 112-122 (2008).

\section{Acknowledgements}

E.C. and G.J.F.D. acknowledge FAPESP (2011/22379-6, 2012/09719-5 and 2016/

12666-1) and CNPq (150025/2017-3) for the financial support, Florian Molton and
Dr Carole Duboc for the EPR measurements, Koiti Araki, Jorge da Silva Shinohara and the LQSN group for the Vis-NIR reflectance and polarised Raman spectra, and Prof. Juliana Fonseca de Lima for her precious help. L.L.-Q. and J.C. thank the LabEx Arcane (ANR-11-Labx-003-01) for financial support. We thank the EPSRC UK Nationa Crystallography Service at the University of Southampton for the collection of the crystallographic data. S.R.P.S. thanks the UGPN for a travel award to visit São Paulo for the research conducted.

\section{Author contributions}

E.C. and G.J.-F.D. designed the project. E.C. synthesised NDI-py and MOF-CoNDI-py-2 and did all the spectroscopic characterisations. K.D.G.I.J., D.C.C. and S.R.P.S. prepared all electronic devices and performed the electrical characterisations. G.J.C. and R.I.W. solved the single-crystal structure of MOF-CoNDI-py-2. L.L.-Q. and J.C. performed the electrochemical and EPR analysis. All authors contributed to the text.

\section{Additional information}

Supplementary Information accompanies this paper at https://doi.org/10.1038/s41467017-02215-7.

Competing interests: The authors declare no competing financial interests.

Reprints and permission information is available online at http://npg.nature.com/ reprintsandpermissions/

Publisher's note: Springer Nature remains neutral with regard to jurisdictional claims in published maps and institutional affiliations.

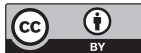

Open Access This article is licensed under a Creative Commons Attribution 4.0 International License, which permits use, sharing, adaptation, distribution and reproduction in any medium or format, as long as you give appropriate credit to the original author(s) and the source, provide a link to the Creative Commons license, and indicate if changes were made. The images or other third party material in this article are included in the article's Creative Commons license, unless indicated otherwise in a credit line to the material. If material is not included in the article's Creative Commons license and your intended use is not permitted by statutory regulation or exceeds the permitted use, you will need to obtain permission directly from the copyright holder. To view a copy of this license, visit http://creativecommons.org/ licenses/by/4.0/.

(c) The Author(s) 2017 